\section{INHIBITION OF INDUCIBLE NITRIC OXIDE SYNTHASE AMELIORATES RAT LUNG ALLOGRAFT REJECTION}

Recently, the inducible isoform of nitric oxide synthase has been shown to be an important immunomodulation molecule in allograft rejection. We have observed the production of nitric oxide during rejection and the effect of nitric oxide synthase inhibition on allograft rejection in a rat lung transplant model. Rat left lung allotransplants were performed in two strain combinations: brown Norway-to-F344 (major histocompatibility complex incompatible); and Lewis-to-F344 (minor loci incompatible) as severe and mild rejection models respectively. Syngeneic F344-to-F344 transplants were performed as a negative control. Nitric oxide production during rejection was determined by measuring the recipient's serum nitrite/nitrate levels as a stable end product of nitric oxide. The progression of rejection was evaluated radiographically and the grade of rejection was determined histologically. After operation, recipients of allotransplantation were randomly divided into two groups and received either aminoguanidine (200 $\mathrm{mg} / \mathrm{kg}$, intraperitoneal every 6 hours), a potent inducible nitric oxide synthase inhibitor, or normal saline treatment. The levels of serum nitrite and nitrate in recipients increased in the early phase of rejection in both allotransplant combinations. However, in the terminal phase of rejection, the serum nitrite/nitrate level decreased significantly compared with the peak leveI in the brown Norway-to-F344 recipients. The serum nitrite/ nitrate levels in the syngeneic transplant recipients were normal during the entire observation period. In aminoguanidine-treated animals, serum nitrite/nitrate levels remained normal in both allograft combinations. Significant suppression of rejection in aminoguanidine-treated recipients was observed histologically and radiographically in comparison with untreated recipients in the brown Norway-to-F344 combination. In the Lewis-to-F344 combination, aminoguanidine treatment significantly ameliorated histologic rejection but did not affect radiologic appearance. We therefore conclude nitric oxide is produced during early allograft rejection and may prove to be a marker and mediator of early rejection. The inhibition of inducible nitric oxide synthase results in significant reduction in rat lung allograft rejection. (J Thorac CARdiovasC SuRg 1995;110: 1449-60)

Takeshi Shiraishi, MD ${ }^{a}$ (by invitation), Steven R. DeMeester, MDa (by invitation), Neil K. Worrall, $\mathrm{MD}^{\mathrm{a}}$ (by invitation), Jon H. Ritter, $\mathrm{MD}^{\mathrm{b}}$ (by invitation), Thomas P. Misko, $\mathrm{PhD}^{\mathrm{c}}$ (by invitation),

T. Bruce Ferguson, Jr., MD, FACS, ${ }^{a}$ Joel D. Cooper, MD, FACS, FRCS $(C),{ }^{a}$ and G. Alexander Patterson, MD, FACS, FRCS(C), ${ }^{a}$ St. Louis, Mo.
From the Division of Cardiothoracic Surgery, ${ }^{a}$ Department of Surgery, Department of Surgical Pathology, ${ }^{b}$ Washington University School of Medicine, Barnes Hospital, St. Louis, Mo., and Department of Molecular Pharmacology, ${ }^{c}$ Searle Research \& Development, Monsanto Company, St. Louis, Mo.

Supported by National Institute of Health grants 1 R01 HL41281 and 5 Rot HL41943.
Read at the Seventy-fifth Annual Meeting of The American Association for Thoracic Surgery, Boston, Mass., April 23-26, 1995.

Address for reprints: G. Alexander Patterson, MD, Professor of Surgery, One Barnes Hospital Plaza, Suite 3108 Queeny Tower, St. Louis, MO 63110.

Copyright (C) 1995 by Mosby-Year Book, Inc.

$0022-5223 / 95 \$ 5.00+0 \quad \mathbf{1 2 / 6 / 6 6 3 3 6}$ 
ung transplantation is now recognized as a therLapeutic option for a variety of end-stage pulmonary diseases. Despite its increasing application, a variety of problems continue to cause significant morbidity and mortality. Acute lung allograft rejection develops to a variable degree in virtually all transplant recipients within the first 4 weeks after the operation. ${ }^{1-4}$ Although acute rejection is rarely fatal, it does increase morbidity. Furthermore, chronic rejection, the commonest cause of late mortality, is related to the frequency and cause of acute rejection episodes. Although much is known about the cellular alterations associated with allograft rejection, investigations directed at the molecular mechanisms of the rejection process may foster development of more precise strategies of rejection prophylaxis.

Nitric oxide (NO), a toxic atmospheric gas, is now known to exist in tissues as the smallest biologic product of mammalian cells. NO is synthesized from the amino acid L-arginine by a family of enzymes, the NO synthases. The constitutive isoform releases small quantities of NO for short periods in response to receptor or physical stimulations. NO derived from the constitutive isoform of NO synthase acts as a physiologic regulator by relaxing vascular smooth muscle $^{5}$ or by functioning as a neurotransmitter. ${ }^{6}$ The inducible isoform of NO synthase can be expressed in several cell types, including activated macrophages and endothelial cells. Once expressed, the inducible isoform synthesizes larger quantities of NO for longer time periods.

Recently, inducible NO has been found to play an important role in immunologic cellular interactions. ${ }^{7-9}$ Increased production of NO has been demonstrated during acute allograft rejection, ${ }^{10-13}$ which suggests that NO may play an important role in the in vivo response to allogeneic tissue. The role of NO in allograft rejection is still unclear; however, in vitro experiments demonstrated a possible role in promoting lymphocyte proliferation. ${ }^{14-16}$ Worrall and associates ${ }^{13}$ have recently demonstrated that NO synthase is induced during cardiac allograft rejection and that selective inhibition of the inducible isoform significantly attenuated cardiac allograft rejection in the rat.

The present study was undertaken to determine if NO was produced during lung allograft rejection and to determine if selective inhibition of the inducible isoform of NO synthase with aminoguanidine, a selective inhibitor of the inducible isoform, ${ }^{17,18}$ would ameliorate the rejection process.

\section{Materials and methods}

Animals. Inbred, male, specific-pathogen-free brown Norway (BN: RT1 ${ }^{\mathrm{n}}$ ), Lewis (LEW: RT1 ${ }^{1}$ ), and F344 $\left(\mathrm{RT} 1^{\mathrm{lvl}}\right)$ rats, weighing 250 to $300 \mathrm{gm}$, were purchased from Harlan Sprague-Dawley (Indianapolis, Ind.). The animals were given humane care in compliance with the "Principles of Laboratory Animal Care" formulated by the National Society for Medical Research and the "Guide for the Care and Use of Laboratory Animals" prepared by the National Academy of Sciences and published by the National Institutes of Health (NIH Publication No. 86-23, revised 1985).

Transplantation. Lung allotransplantation was performed in an RT1 (major histocompatibility complex) incompatible donor-recipient combination (BN donor into F344 recipients), as well as in a combination incompatible for minor loci (LEW to F344), to severe and mild rejection models, respectively. Additionally, syngeneic transplants were performed in an F344 to F344 combination. F344 rats were used as recipients in all groups. The orthotopic left lung transplantation procedure used in this study was a modification of the "cuff" technique. ${ }^{19}$ In brief, the donor rats were anesthetized with intraperitoneal administration of pentobarbital $(20 \mathrm{mg} / \mathrm{kg})$ after intramuscular injection of atropine $(0.25 \mathrm{mg} / \mathrm{kg})$ and ketamine $(25 \mathrm{mg} / \mathrm{kg})$. After intravenous administration of heparin $(1000 \mathrm{U} / \mathrm{kg})$ and median sternotomy with the rat mechanically ventilated with room air, both lungs were flushed with cold $\left(1^{\circ} \mathrm{C}\right)$ low potassium dextran-1\% glucose (LPDG) solution at $20 \mathrm{~cm} \mathrm{H}_{2} \mathrm{O}$ pressure. The heart-lung block was excised with the lungs inflated at end tidal volume and immersed in cold $\left(1^{\circ} \mathrm{C}\right)$ low potassium dextran-1\% glucose solution until implantation. Recipient animals were intubated and anesthesia was maintained by mechanical ventilation with halothane and oxygen by the use of a small-animal ventilator (Harvard Rodent Ventilator, South Natick, Mass.). After left pneumonectomy was performed, the pulmonary artery and vein were anastomosed with the aid of polyethylene cuffs with internal diameters of $1.65 \mathrm{~mm}$ and $2.20 \mathrm{~mm}$, respectively, and the left main bronchus was anastomosed with 8-0 Prolene running suture (Ethicon, Inc., Somerville, N.J.).

After left lung transplantation in rats, radiographic assessment of the graft can be obscured by the right lung. Therefore, a posteaval lobectomy on the recipient right lung was performed immediately after implantation in each recipient so as to facilitate radiographic assessment of the left lung allograft. After the chest wall was closed, a silicone tube was placed intraperitoneally, tunneled subcutaneously, and brought out posteriorly behind the animal's head to provide access for regular intraperitoneal drug administration. Animals were given supplemental oxygen for 24 hours after the operation. No immunosuppressive medication was used in this study.

Rats having acute allograft dysfunction as evidenced by abnormalities on the chest roentgenogram immediately after implantation were excluded from the analysis on the basis of technical failure.

Experimental groups. The total number of transplants was 17 in the BN-to-F344 group, 16 in the LEW-to-F344 group, and 7 in the syngeneic group. Allograft recipients 
The Journal of Thoracic and

Cardiovascular Surgery

Shiraishi et al. 1451

Volume 110, Number 5

Table I. Experimental groups

\begin{tabular}{|c|c|c|c|c|}
\hline Strain combination & Aminoguanidine treatment & Graft ischemic time (min) & Day of planned death & $n$ \\
\hline \multirow[t]{2}{*}{$\mathrm{BN}$ to $\mathrm{F} 344$} & Yes & $109.00 \pm 7.60$ & \multirow[t]{2}{*}{7} & 9 \\
\hline & No & $108.78 \pm 5.24\} \mathrm{NS}$ & & 8 \\
\hline \multirow[t]{2}{*}{ LEW to F344 } & Yes & $105.88 \pm 5.82$ & \multirow[t]{3}{*}{14} & 8 \\
\hline & No & $108.00 \pm 4.81\} \mathrm{NS}$ & & 8 \\
\hline F344 to F344 & No & $109.43 \pm 7.04$ & & 7 \\
\hline
\end{tabular}

Aminoguanidine treatment was performed by injecting a $200 \mathrm{mg} / \mathrm{kg}$ dose of aminoguanidine hemisulfate intraperitoneally through the implanted catheter every 6 hours until the rat was put to death. The first administration was performed 6 hours after implantation. Control group in each combination and syngeneic group (F344 to F344) received same amount of normal saline solution in the same manner. NS, Not significant.

from each strain combination were randomized into two groups and were treated with either aminoguanidine or normal saline solution (Table I). The aminoguanidinetreated group received $1.5 \mathrm{ml}$ of normal saline solution containing a $200 \mathrm{mg} / \mathrm{kg}$ concentration of aminoguanidine hemisulfate salt (Sigma Chemical Company, St. Louis, Mo.) with a $0.5 \mathrm{mg} / \mathrm{kg}$ dose of gentamicin (aminoguanidine-treated group) intraperitoneally through the implanted catheter every 6 hours until the rat was put to death. The control group received $1.5 \mathrm{ml}$ of normal saline solution containing only a $0.5 \mathrm{mg} / \mathrm{kg}$ concentration of gentamicin (untreated group) intraperitoneally every 6 hours in a similar fashion. All recipients of syngeneic transplants $(n=7)$ received normal saline solution with a $0.5 \mathrm{mg} / \mathrm{kg}$ dose of gentamicin intraperitoneally in the same manner. The first administration in all groups was performed 6 hours after implantation.

Serum nitrite/nitrate assay. Recipient NO production in each group was determined by measuring serum nitrite/ nitrate levels, which are stable end products of $\mathrm{NO}$ metabolism, ${ }^{10,20}$ at serial time points after transplantation. Blood samples for this assay were obtained from the tail artery on days 4 and 7 in the recipients of BN allografts and on days 7, 11, and 14 in the LEW allograft recipients. Blood samples for this assay were taken from the syngeneic group on days 4, 7, and 14. Additionally, the blood samples from three normal F344 rats served as controls. In this study, serum nitrite/nitrate levels were determined by the recently described fluorometric assay reported by Misko and colleagues. ${ }^{21}$ In brief, serum samples were first filtered through a 10,000 molecular weight (Mw) cutoff filter to remove the hemoglobin resulting from cell lysis. The serum nitrate was converted to nitrite by the action of nitrate reductase by incubating with 40 reduced nicotinamide adenine dinucleotide phosphate (NADPH), $40 \mu \mathrm{mol} / \mathrm{L}$ (to initiate the reaction), and $14 \mathrm{mU}$ of nitrate reductase (Sigma) in a final volume of 50 $\mu \mathrm{l}$ of Tris buffer $(20 \mathrm{mmol} / \mathrm{L}), \mathrm{pH} 7.6$. The reaction was terminated after 5 minutes at $20^{\circ} \mathrm{C}$ by dilution with $50 \mu \mathrm{l}$ of water followed by the addition of 2,3-diaminonaphthalene for determination of nitrite. 2,3-Diaminophthalene reacts with nitrite under acidic conditions to form 1- $(H)$ naphthotriazole, a fluorescent product. Formation of 1- $(H)$-naphthotriazole was measured with a Pandex fluorescent plate reader (IDEXX Laboratories Inc., Westbrook, Maine) with excitation at $365 \mathrm{~nm}$ and emission read at $450 \mathrm{~nm}$ with a gain setting of $100 \%$. Nitrite levels in the samples were then calculated by first subtracting the value of the enzyme blank (i.e., nitrate reductase plus
NADPH) from the experimental value and then calculating the value using a standard curve for nitrite to which NADPH has been added. Plasma volumes of $10 \mu \mathrm{l}$ or less routinely give a linear response in this assay.

Radiographic analysis. Transplanted lungs were assessed radiographically each day in the recipients of $B N$ allografts and every other day in animals with LEW allografts during spontaneous ventilation with halothane inhalation for sedation. The first examination was performed on day 1 after transplantation. Anteroposterior chest roentgenograms were taken with the rat in a slightly left anterior oblique position so as to provide maximum exposure of the transplanted left lung. Results of each chest $x$-ray film were graded according to a previously published aeration score ${ }^{22}$ by a blinded observer (score $0=$ opaque lung up to score $6=$ normal-appearing lung). Recipients of syngeneic transplants did not undergo radiographic assessment, because the purpose of this group was to evaluate the production of $\mathrm{NO}$ in association with surgical stress and ischemia-reperfusion injury.

Histologic studies. Recipients of $\mathrm{BN}$ allografts were put to death on day 7 , by which time complete graft rejection would be expected histologically and radiographically in this severely mismatched combination (Shiraishi and associates, unpblished data). Our preliminary study in LEW allograft recipients demonstrated that the histologic peak of rejection was on day $14^{23}$; however, complete rejection in this combination is less clearly defined, and occasionally spontaneous recovery occurs 3 weeks after transplantation. ${ }^{24}$ Therefore, those recipients were put to death on day 14 . At their death, $10 \%$ buffered formaldehyde solution was infused into the trachea at a hydrostatic pressure of $20 \mathrm{~cm} \mathrm{H}_{2} \mathrm{O}$. The heart and lungs were then removed en bloc from the thoracic cage. Allografts were divided in a coronal plane through the main bronchus so as to produce two equal sections and maximal longitudinal bronchial length. Paraffin sections were stained with hematoxylin and eosin for examination with a light microscope. Acute rejection was evaluated histologically and assigned a rejection score based on the clinical international working formulation: IWF grade $\mathrm{A} 0$, no significant abnormality; grade A1, scattered infrequent perivascular mononuclear infiltration; grade A2, frequent perivascular mononuclear infiltration; grade $\mathrm{A} 3$, extension of mononuclear infiltration into alveolar septum/ spaces; and grade A4, diffuse perivascular, interstitial, and air space infiltration of mononuclear cells. ${ }^{25}$ In addition, allografts were also evaluated according to the scoring system devised by Prop and coworkers ${ }^{26}$ for rat lung 

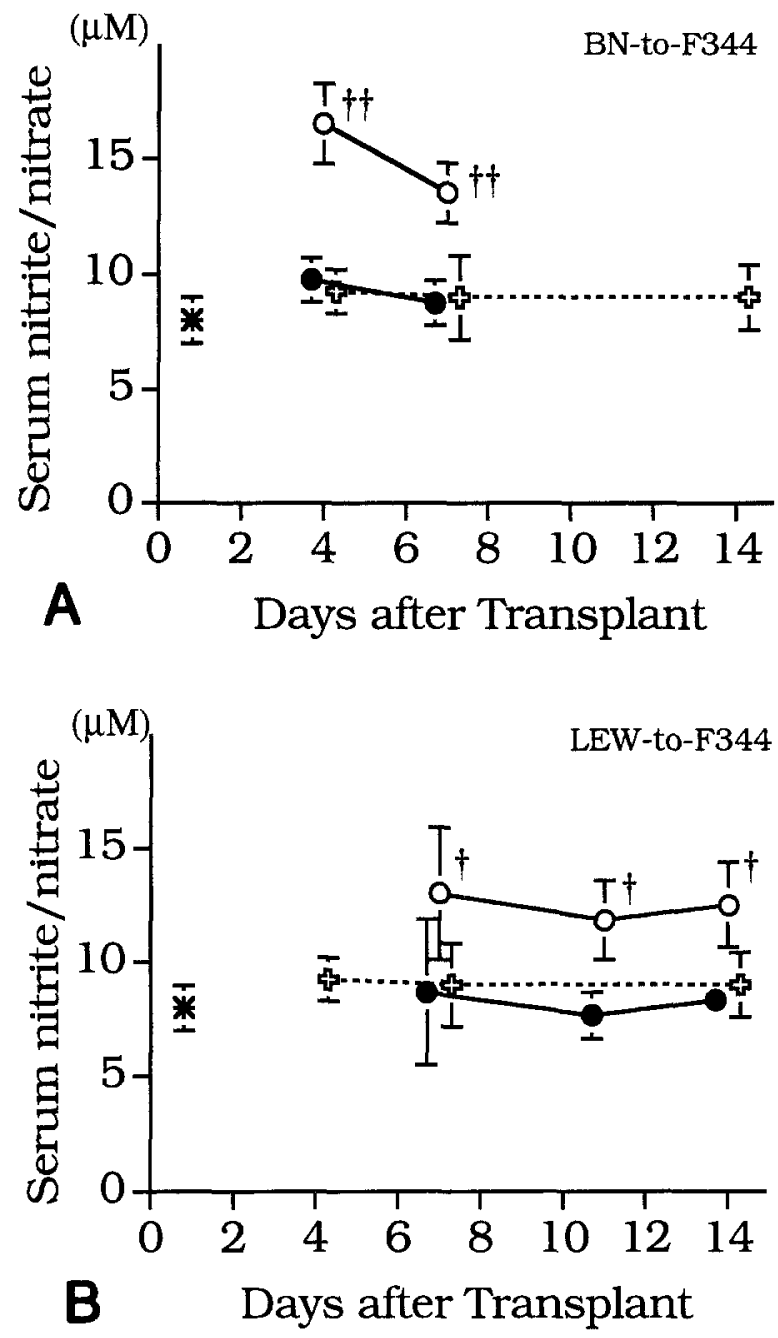

Fig. 1. Change of serum nitrite/nitrate levels in the F344 recipients of $\mathrm{BN}$ and $\mathrm{LEW}$ allografts. $\mathrm{A}, \mathrm{BN}-$ to-F344: The levels of serum nitrite/nitrate in aminoguanidine-treated (closed circle, $n=4$ ) or untreated (open circle, $n=4$ ) recipients of $\mathrm{BN}$ allografts and recipients of F344 syngeneic grafts: (open cross, $n=4$ ) were determined on the postoperative days indicated. B, LEW-to-F344: The levels of serum nitrite/nitrate in aminoguanidine-treated (closed circle, $n=$ 6), untreated (open circle, $n=6$ ) recipients of LEW allografts and recipients of syngeneic graft (open cross, $n=4$ ) were determined on the postoperative days indicated. Each point represents the mean \pm standard deviation. The symbol * indicates the mean \pm SEM normal level in F344 rats $(n=$ 3 ). The symbol + indicates that values for untreated recipients are significantly different from those of treated and syngeneic recipients. $\uparrow p<0.05 ; \dagger \uparrow p<0.01$.

allograft rejection: grade 0 -latent phase, no signs of rejection; grade 1 - vascular phase; perivascular and bronchus-associated lymphoid tissue infiltration; grade 2 -alveolar phase, alveolar infiltration; grade 3-destruction phase, graft destruction. Both histologic assessments were performed by a blinded pathologist (J.H.R.). This histologic assessment was not performed on the syngeneic grafts.

Statistical analysis. The data were expressed as mean \pm standard deviation. Intergroup comparisons of radiographic and histologic parameters were analyzed by the Mann-Whitney $U$ test. Serum nitrite/nitrate levels were compared by two-way analysis of variance. Multiple comparisons were made using the Tukey highest significant difference post-hoc test. Differences were considered significant if the $p$ value was less than 0.05 .

\section{Results}

All animals survived the transplant procedure. One BN allograft recipient treated with aminoguanidine died of empyema on postoperative day 4 and was excluded from subsequent analysis. Early allograft function was satisfactory in all animals; therefore no animals were excluded on the basis of radiographic score. Total allograft ischemic time did not differ significantly between aminoguanidinetreated and untreated recipients of both the $\mathrm{BN}$ and LEW allograft groups (Table I).

\section{Serum nitrite/nitrate level}

F344-to-F344. Serum nitrite/nitrate levels in the normal F344 rats was $8.0 \pm 1.0 \mu \mathrm{mol} / \mathrm{L}(n=3)$. The serum nitrite/nitrate levels of syngeneic recipients did not differ from normal values (Fig. 1).

$B N-t o-F 344$. In the BN allograft group, the serum nitrite/nitrate levels of the untreated recipients were significantly higher than those of the aminoguanidine-treated recipients or syngeneic recipients on days 4 and 7 . The value on day 4 was significantly higher than on day 7 in the untreated recipients. Normal values were found in the aminoguanidinetreated recipients at each time point (Fig. 1, $A$ ).

LEW-to-F344. In the LEW allograft group, the serum nitrite/nitrate levels of the untreated recipients were significantly higher than those of the aminoguanidine-treated recipients on days 7,11 , and 14. Values for aminoguanidine-treated recipients were within normal limits at each time point (Fig. 1, B).

\section{Radiographic findings}

$B N$-to-F344. All recipients of $\mathrm{BN}$ allografts showed excellent aeration of the transplanted left lung during the first 4 days after operation. From day 5 until the rats were put to death at day 7 , there was a progressive decrease in aeration score in the untreated group, and on day 7 all eight untreated recipients had a completely opaque chest $\mathrm{x}$-ray film. Aminoguanidine-treated recipients had less opacifi- 
cation of the lung graft between days 5 and 7. A significant difference was present at days 6 and 7 between untreated and treated recipients $(1.75 \pm$ 0.87 versus $4.08 \pm 1.39$ on day $6, p=0.017 ; 0.9 \pm$ 0.74 versus $4.17 \pm 1.40$ on day $7, p=0.006$ ) (Fig. 2, $A$ and Fig. 3).

LEW-to-F344. All recipients of LEW allografts had clear chest $\mathrm{x}$-ray films until being put to death. There were no significant differences between treated and untreated groups at each time point through their planned deaths (Fig. 2, B).

\section{Pathologic findings}

$B N$-to-F344. Macroscopic inspection under mechanical ventilation at the time of sacrifice revealed that all eight untreated $\mathrm{BN}$ allograft recipients showed significant heparinization, scattered hemorrhage, massive atelectasis, and complete consolidation without aeration whereas all nine allografts in the treated group were well ventilated. In contrast, the grafted lungs in aminoguanidine-treated recipients appeared similar to the contralateral native lungs (Fig. 4). The radiographic findings of less rejection in the aminoguanidine-treated group were confirmed, histologically (Fig. 5). Significant improvements in the histologic stage of rejection between untreated and treated groups were found by means of both the clinical and the Prop scoring systems in the recipients of BN allografts $(3.75 \pm$ 0.54 versus $2.61 \pm 0.22$ in IWF grade, $p=0.0015$; $2.63 \pm 0.74$ versus $1.33 \pm 0.35$ in the Prop score, $p=$ 0.0042) (Fig. 6, $A$ ).

LEW-to-F344. All recipients of LEW allografts had well-ventilated lungs. According to the IWF grading system, aminoguanidine-treated recipients had less rejection than the untreated group $(2.5 \pm 0$ versus $2.25 \pm 0.27$, in untreated and treated groups, respectively, $p=0.025$ ). There was no significant difference, however, when the groups were compared by means of Prop's scoring system (Figs. 6, $B$ and 7).

\section{Discussion}

Recently NO has been found to play a fundamental role in host immune defense. ${ }^{8}$ Activated macrophages can be induced to express the inducible isoform of NO synthase, thereby oxidatively metabolizing the amino acid L-arginine to produce NO, citrulline, nitrite, and nitrase. This pathway was found to be enhanced in the presence of lipopolysaccharide and the cytokines interleukin-2, interferon- $\gamma$, and tumor necrosis factor- $\alpha .{ }^{27}$ NO produced by macrophages has been demonstrated to respond
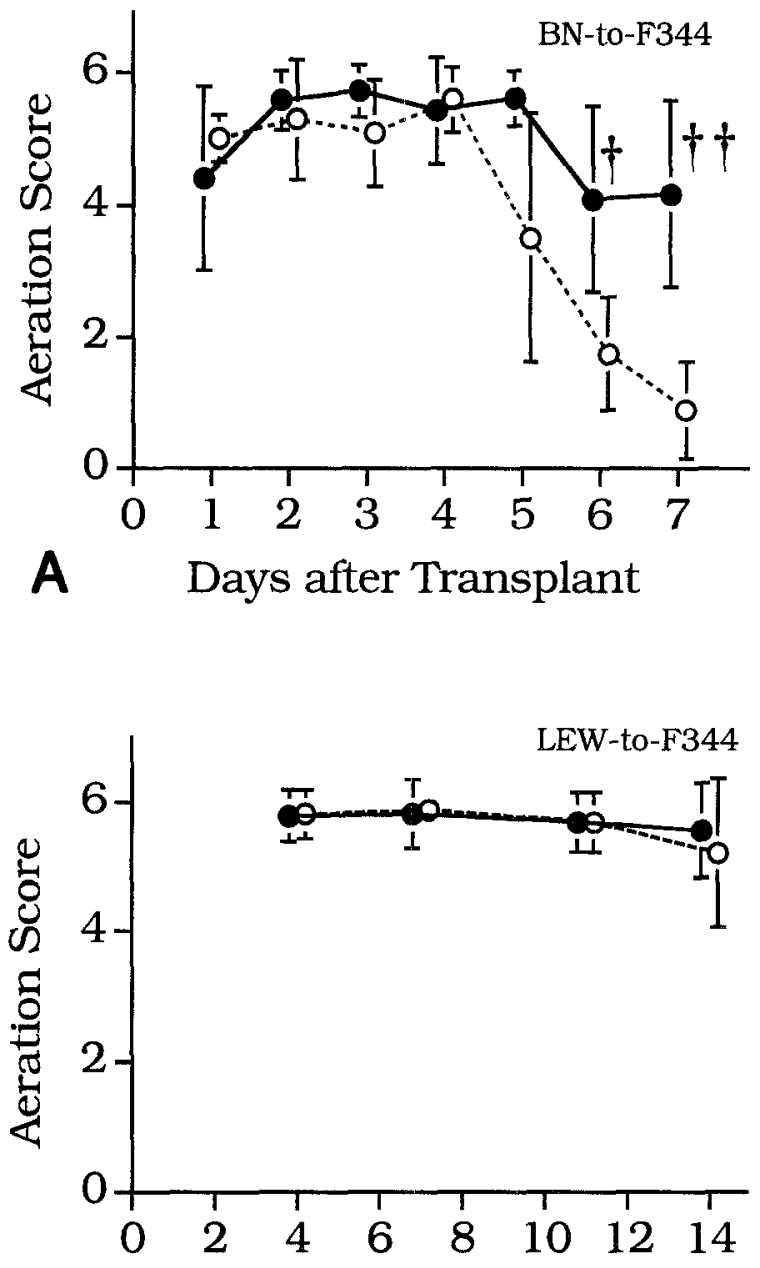

B Days after Transplant

Fig. 2. A and B, Change of chest $x$-ray findings in the F344 recipients of BN and LEW allografts Aeration score indicates 0 for opaque to 6 for normal-appearing lung. Closed circle and open circle demonstrate aminoguanidinetreated and untreated recipients, respectively. Each point represents the mean \pm standard deviation. The symbol $\uparrow$ indicates values that are significantly different in intergroup comparison. $\uparrow p<0.05 ; \uparrow \uparrow p<0.01$.

as a nonspecific defense mechanism against a variety of pathogens ${ }^{28-31}$ and tumor cells. ${ }^{32-34}$ The biochemical basis of this cytostatic/cytotoxic function induced by NO is thought to be a result of nitrosylation and inhibition of key enzymes involved in the respiratory cycle and the synthesis of deoxyribonucleic acid in the target cells. ${ }^{8,35,36}$

The production of NO during allograft rejection has been demonstrated in several experimental 


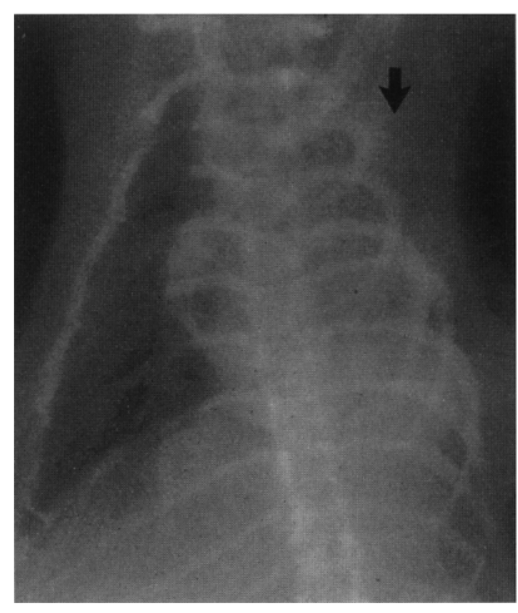

Untreated

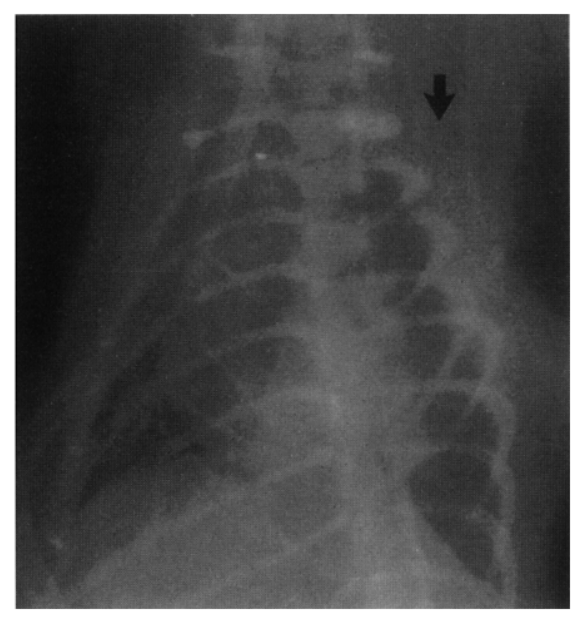

Treated

Fig. 3. Chest $x$-ray films of F344 recipients of BN allografts. Representative chest $x$-ray (anteroposterior view) photographs of aminoguanidine-untreated (left) and treated (right) recipients of BN allografts 7 days after transplantation. Black arrows indicate transplanted left lungs. Transplanted lungs of untreated recipients were completely opaque (aeration score $=0$ ), whereas treated animals demonstrated only minimal changes in infiltration (aeration score $=5$ ).

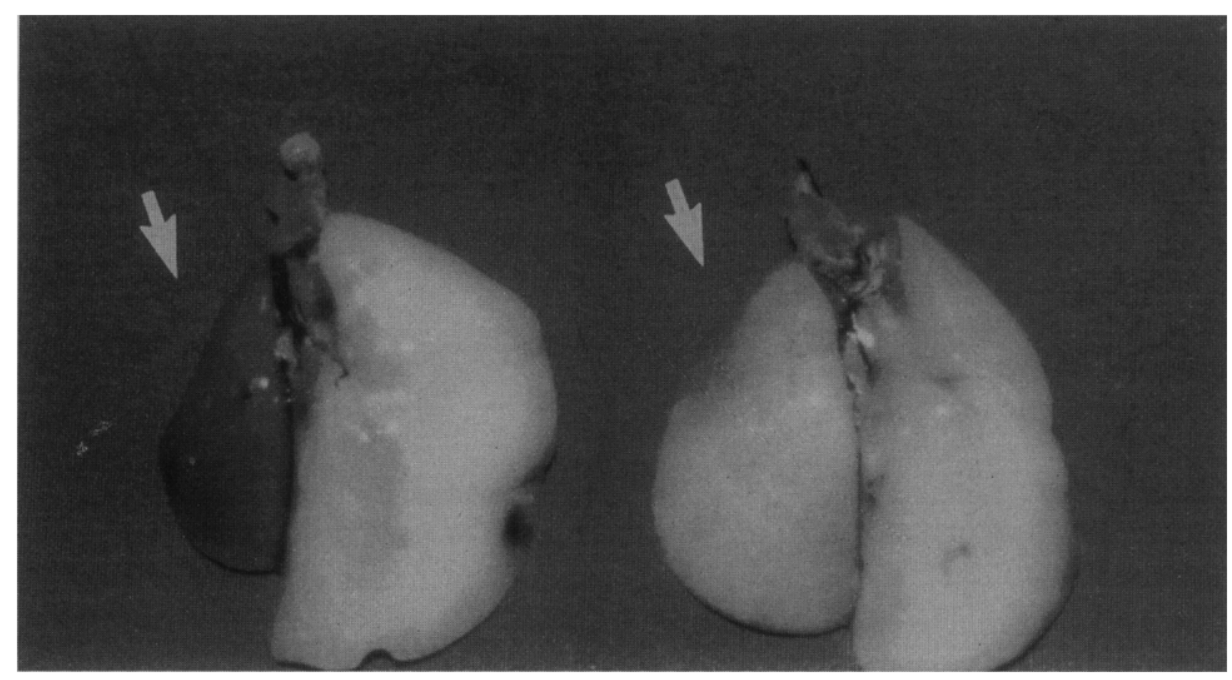

\section{Untreated Treated}

Fig. 4. Gross view of $\mathrm{BN}$ lung allografts on day 7 after transplantation. Gross examination of the transplanted left lung (arrow) showed significant signs of heparinization with massive atelectasis in the untreated recipient (left), whereas the aminoguanidine-treated recipient appeared similar to the native contralateral lung (right).

models. Increased NO production, as measured by elevated serum nitrite/nitrate levels, was found in the recipients of rejecting rat heart, liver, small bowel, and bone marrow allografts. ${ }^{10,13}$ Similarly, allosensitized rat-sponge-matrix allograft-infiltrating cells were found to produce higher quantities of NO than did cells infiltrating syngeneic grafts in murine and rat models. ${ }^{27,37-40}$ Direct evidence of $\mathrm{NO}$ in 

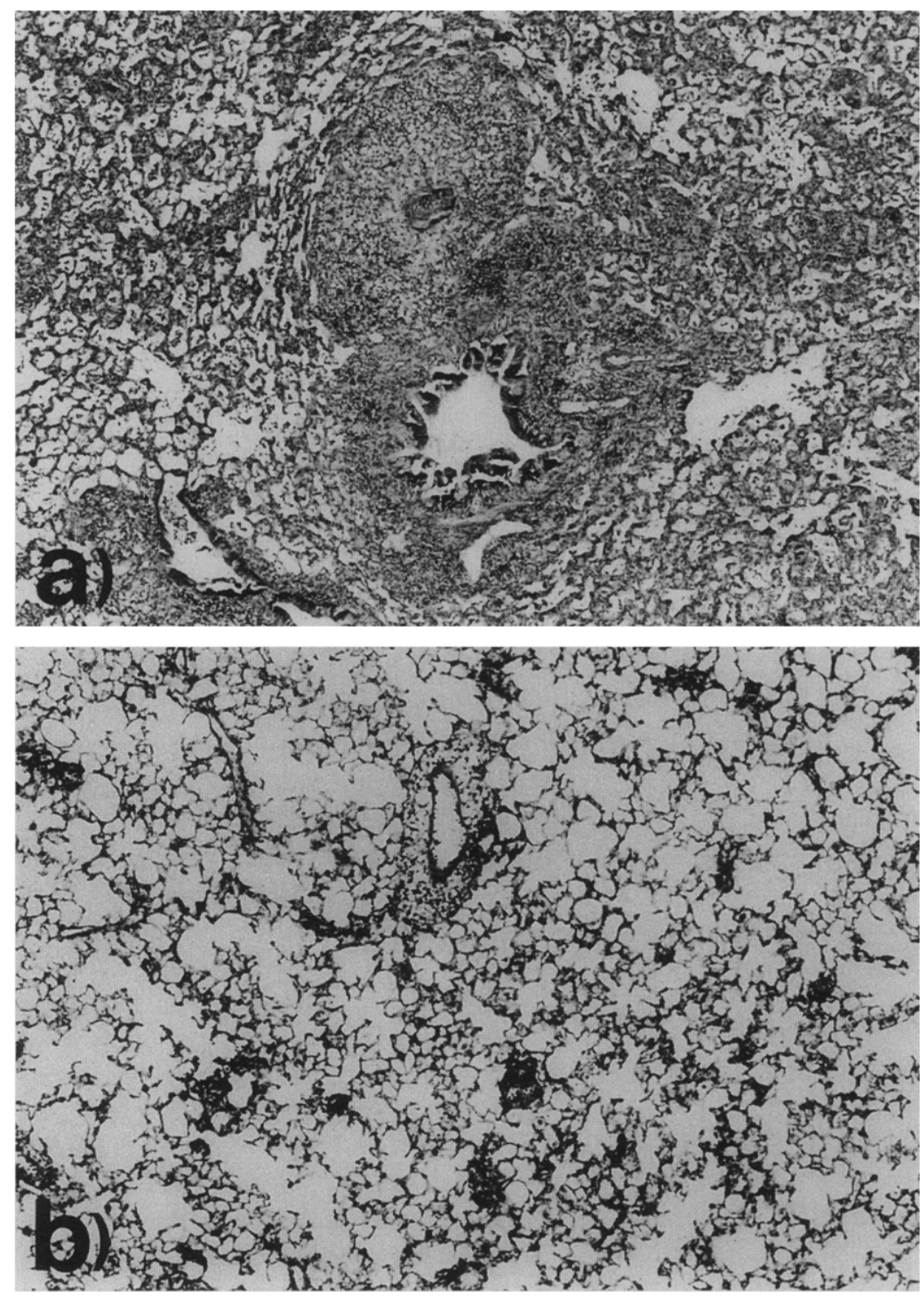

Fig. 5. Representative photomicrographs of BN allografts to F344 recipients at day 7 after transplantation ( $\times 400)$. a, Aminoguanidine-untreated recipient: severe rejection with confluent, destructive infiltrates (IWF grade 4; Prop score 3). b, Aminoguanidine-treated recipient: mild to weak rejection; perivascular infiltrates are present without extension into alveoli (IWF grade 2 to 3, Prop score 1).

cardiac allografts has been obtained by electron paramagnetic resonance spectroscopy, which detected the signals for nitrosylferrohemoglobin and nonheme iron-dinitrosyl complexes in the allograft tissue. $^{12,13}$ The increased levels of serum nitrite and nitrate in allograft recipients can be suppressed by treatment with the immunosuppressive drugs FK $506^{38}$ and cyclosporine A. $^{10}$ Moreover, when the immunosuppressive therapy was terminated and histologic evidence of recurrent rejection was found, elevated serum nitrite/nitrate levels were detected.
These data seem to suggest that NO is produced in association with rejection and may have some important role in the rejection process. However, the mechanism of NO production and the role of NO in allograft rejection is unclear. Several reports demonstrate NO mediated in vitro T-lymphocyte suppression. NO produced by concanavalin A-stimulated rat splenocytes is associated with a profound suppression of lymphocyte proliferation. Furthermore, inhibition of NO production with L- $N$-nitroso$\mathrm{N}$-methylalanine markedly promotes this mitogenic 

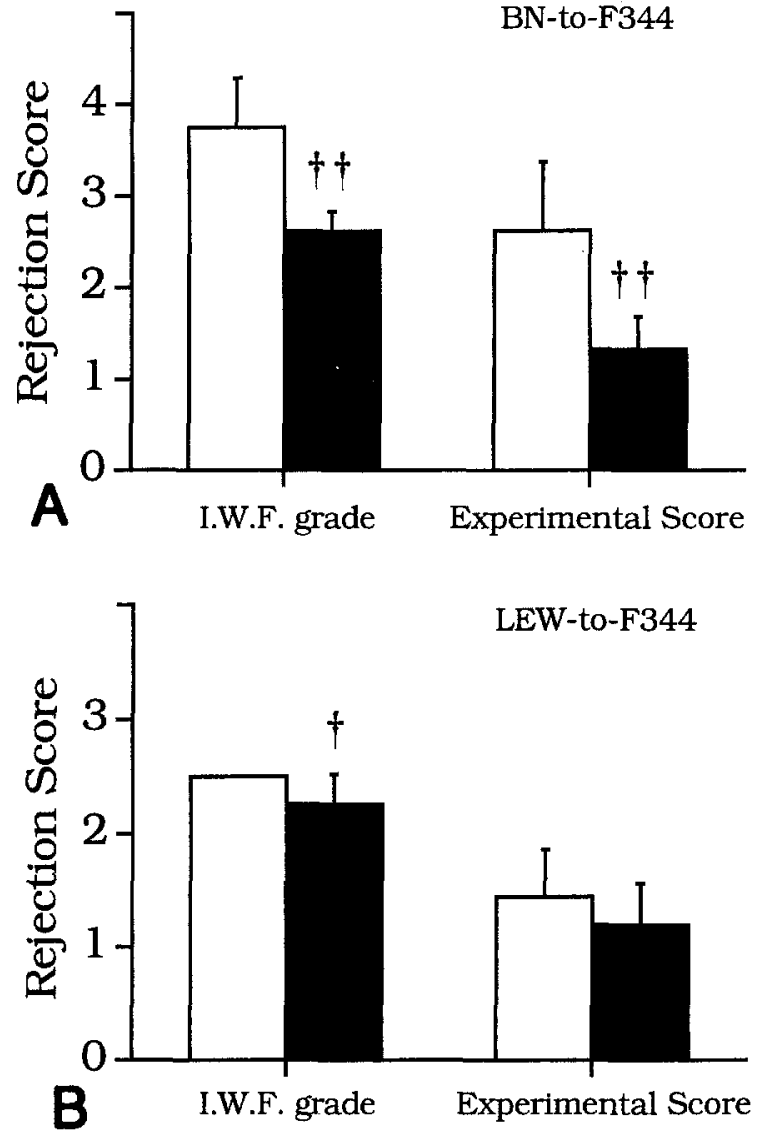

Fig. 6. Rejection scores of BN and LEW allografts when the rats were killed. BN lungs 7 days after transplantation to F344 recipients (A) and LEW lungs 14 days after transplantation to F344 recipients (B). Black bars and white bars indicate the allografts of aminoguanidinetreated and untreated recipients, respectively. Rejection was evaluated histologically on the basis of perivascular infiltration according to the IWF (IWF grade A0, no significant abnormality; grade A1, scattered infrequent perivascular mononuclear infiltration; grade $\mathrm{A} 2$, frequent perivascular mononuclear infiltration; grade A3, extension of mononuclear infiltration into alveolar septum/ spaces; grade A4, diffuse perivascular, interstitial, and air space infiltration of mononuclear cells). Allografts were also evaluated according to the Prop scoring system for rat lung allograft rejection (grade 0 -latent phase: no signs of rejection; grade 1-vascular phase: perivascular and bronchus-associated lymphoid tissue infiltration; grade 2-alveolar phase: alveolar infiltration; grade 3-destruction phase: graft destruction). All values are expressed as mean \pm standard deviation of the mean. The symbol $\dagger$ indicates values that are significantly different in intergroup comparison. $\dagger p<0.05 ; \dagger p<0.01$. response to concanavalin $\mathrm{A}^{41} \mathrm{NO}$ has also been shown to down-regulate certain macrophage functions, including superoxide production, phagocytosis, and protein synthesis. ${ }^{42,43}$ Langrehr, $20,27,39$ Hoffman, ${ }^{40}$ and their associates, using a sponge matrix allotransplant model, demonstrated that $\mathrm{NO}$ production in the rat and mouse splenocyte mixedlymphocyte reaction promoted a profound inhibitory effect on cytotoxic T-lymphocyte induction. These findings seem to suggest a suppressive role for $\mathrm{NO}$ in alloreactivity.

Other studies, however, suggest an immunostimulating capacity of NO. Efron and colleagues ${ }^{14}$ used phytohemagglutinin-stimulated human mononuclear cell culture to describe the ability of NO to promote deoxyribonucleic acid synthesis and thereby enhance lymphocyte proliferation. These data appear at first glance to be paradoxic and contrary to the reports that demonstrate the inhibitory effect of $\mathrm{NO}$ on lymphocyte proliferation. More recently, a similar promotive effect of $\mathrm{NO}$ on lymphocyte proliferation was demonstrated in a murine splenocyte culture model. ${ }^{15}$ In addition, Sugiyama and associates ${ }^{16}$ noted that inhibition of NO synthase by aminoguanidine inhibits cell proliferation without cell death by prolonging the $\mathrm{M}$ phase of the cell cycle in rat liver and human tumor cell lines. Considering these discordant observations on the immunoregulatory roles of NO, it is likely that NO plays multiple roles in allograft rejection.

For the particular effect of NO on transplanted allograft rejection to be elucidated, further experiments using vascularized organ transplant models seem necessary because previous studies have been performed with in vitro cell culture models or nonvascularized "sponge matrix" transplant models. Recently, Worrall and associates ${ }^{13}$ have demonstrated that the inducible isoforme of NO synthase is expressed in the inflammatory cells infiltrating cardiac allografts and that inhibition of the inducible isoform prolonged cardiac allograft survival and improved graft function. This suggests that the net role of $\mathrm{NO}$ is to promote organ rejection.

In the present study, we have demonstrated that NO is produced during lung allograft rejection, and selective inhibition of the inducible isoforme of NO synthase with aminoguanidine significantly attenuated the rejection process. NO production was demonstrated by elevated serum nitrite/nitrate levels in untreated F344 recipients of both "weak mismatched" LEW and "strong mismatched" BN lung allografts. In recipients of both LEW and BN allografts, the levels of serum 

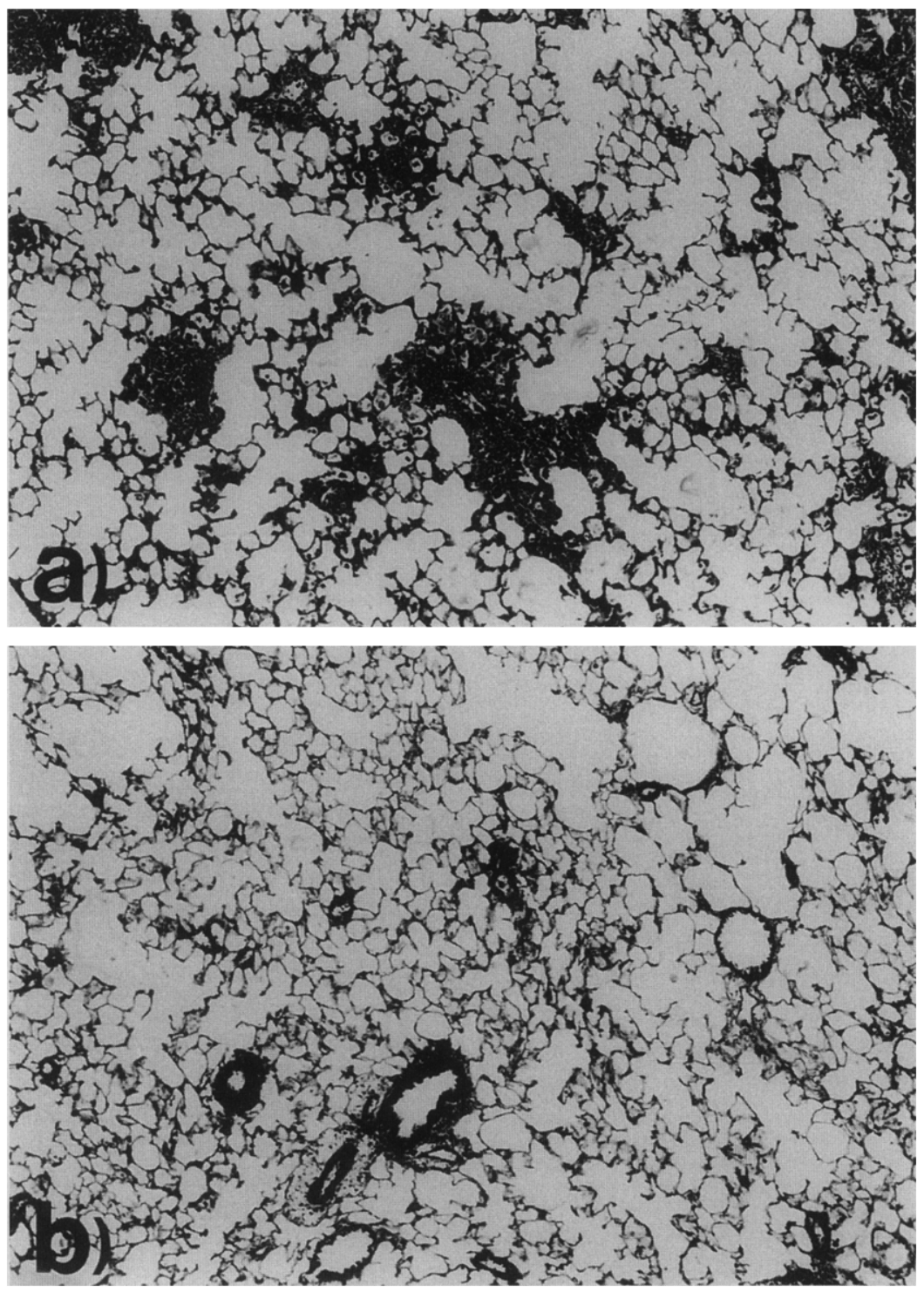

Fig. 7. Representative photomicrographs of LEW allografts to F344 recipients at day 14 after transplantation $(\times 400)$. a, LEW allograft from aminoguanidine untreated recipient: mild to moderate rejection with perivascular lymphoid cuffs and minimal extension to alveoli. b. Treated recipient: slight rejection compared with untreated recipient.

nitrite and nitrate started to increase during the early phases of rejection. Indeed, in the recipients of LEW allografts, elevated levels of serum nitrite and nitrate were detected on day 7 . Yet, we $\mathrm{e}^{23}$ have previously shown that at day 7 this same untreated LEW-to-F344 combination demonstrated a low-grade histologic rejection (IWF grade $=2.06 \pm 0.18$ ). In the recipients of rapidly rejecting $\mathrm{BN}$ allografts, increased serum nitrite/ nitrate levels were detected on day 4 but were signifcantly decreased by day 7 , when complete histologic rejection had occurred. We were not able to observe a similar decrease in serum nitrite/nitrate level in the "weak mismatched" LEW allograft recipients. These data suggest that serum nitrite/nitrate levels may serve as an early marker of rejection. With complete rejection, however, the destruction and thrombosis of microvasculature in the allograft may result in decreased release of NO from the rejecting organ. Aminoguanidine treatment normalized the serum nitrite/nitrate levels in both allotransplant strain combinations throughout the time-course of rejection.

Graft survival studies are generally not performed 
in the rat lung transplantation model because of the complexity of assessing function of the allograft. Consequently, radiographic and histologic analysis have served as the standard parameters in measuring the severity of rejection. Both treated and untreated recipients of "strong mismatched" BN allografts showed excellent radiographic findings until day 4 after transplantation. However, after days 5 to 7 , the aeration score progressively decreased in the untreated group whereas the treated group showed only minimal decrease. These observations correlated well with the histologic rejection scores. Significant differences in rejection scores were observed with both IWF grade and Prop scoring systems. In the group of "weak mismatched" LEW allograft recipients, there was no difference in the $\mathrm{x}$-ray findings between treated and untreated groups. However, the histologic IWF grade was slightly but significantly suppressed in treated recipients.

Although it may be possible that aminoguanidine acted via a different mechanism and the serum nitrite/nitrate levels were merely reflective of the degree of rejection, we believe that it was the inhibition of the inducible isoforme of NO synthase that by aminoguanidine resulted in the lower level of NO. We may hypothesize that the lower graft level of NO resulted in a less severe alloimmune response and led to a delay in rejection in those grafts. Additionally, considering the chemical capacity of $\mathrm{NO}$ as a free radical, there is another possible mechanism for the promotive effect of $\mathrm{NO}$ on the rejection process. It has been demonstrated that nonspecific injury, such as viral infection or ischemia-reperfusion injury, might predispose the allograft to subsequent rejection through up-regulation of class II major histocompatibility complex antigens $^{44}$ or increased local production of cytokines in the allograft ${ }^{45}$ that closely relate to the rejection process. Therefore we hypothesize that NO injures allografts and results in increased alloantigenicity.

We conclude that aminoguanidine suppressed the production of $\mathrm{NO}$ associated with rejection and ameliorated rat lung rejection as a net effect. The role of NO in the rejection process remains unclear. Further immunologic or molecular biologic investigation seems to be necessary to elucidate the mechanism.

We thank Richard B. Schuessler, PhD, for his expertise in the statistical evaluation, Dawn G. Schuessler for preparation of manuscript, and Donna M. Marquart for technical support.

\section{REFERENCES}

1. Grossman RF, Cooper JD. Lung transplantation. Immunol Allergy Clin North Am 1989;9:153-8.

2. Theodore J, Lewinston N. Lung transplantation comes of age. N Engl J Med 1990;322:772-4.

3. Cooper JD, Patterson GA, Grossman R, Maurer J. Double lung transplantation for advanced chronic obstructive lung disease. Am Rev Respir Dis 1989; 139:303-7.

4. Penketh ARL, Higenbottam TW, Hutter J, Coutts C, Stewart S, Wallwork J. Clinical experience in the management of pulmonary opportunitistic infection and rejection of heart-lung transplants. Thorax 1988; 43:762-9.

5. Palmar RMJ, Ashton DS, Moncada S. Vascular endothelial cells synthesize nitric oxide from L-arginine. Nature 1988;333:664-6.

6. Garthwaite J. Glutamine, nitric oxide and cell-cell signalling in the nervous system. Trends Neurosci 1991;14:60-7.

7. Nussler AK, Billiar TR. Inflammation, immunoregulation, and inducible oxide synthase. J Leukoc Biol 1993;54:171-8.

8. Moncada S, Higgs EA. The L-arginine-nitric oxide pathway. N Engl J Med 1993;329:2002-12.

9. Moncada S, Palmar RMJ, Higgs EA. Nitric oxide: physiology, pathophysiology, and pharmacology. Pharmacol Rev 1991;43:109-42.

10. Langrehr JM, Murase N, Markus PM, et al. Nitric oxide production in host-versus-graft and graft-versus-host reactions in the rat. J Clin Invest 1992;90: 679-83.

11. Langrehr JM, Muller AR, Bergonia HA, et al. Detection of nitric oxide by electron paramagnetic resonance spectroscopy during rejection and graft-versushost disease after small-bowel transplantation in the rat. Surgery 1992;112:395-402.

12. Lancaster JR, Langrehr JM, Bergonia HA, Murase N, Simmons RL, Hoffman RA. EPR detection of heme and nonheme iron-containing protein nitrosylation by nitric oxide during rejection rat heart allograft. J Biol Chem 1992;267:10994-8.

13. Worrall NK, Lazenby WD, Misko TP, et al. Modulation of in vivo alloreactivity by inhibition of inducible nitric oxide synthase. J Exp Med 1995;181:63-70.

14. Efron DT, Kirk SJ, Regan MC, Wasserkrug HL, Barbul A. Nitric oxide generation from L-arginine is required for optimal human peripheral blood lymphocyte DNA synthesis. Surgery 1991;110:327-34.

15. Gregory SH, Sagnimeni AJ, Wing EJ. Arginine analogues suppress antigen-specific and non-specific T lymphocyte proliferation. Cell Immunol 1994;153:527-32. 
16. Sugiyama T, Shimada H, Nomura M, Miyamoto K. Aminoguanidine inhibits cell proliferation by prolongation of mitotic phase. Toxicol Lett 1993;69:273-8.

17. Corbett JA, Tilton RG, Chang K, et al. Aminoguanidine, a novel inhibitor of nitric oxide formation, prevents diabetic vascular dysfunction. Diabetes 1992; 41:552-6.

18. Misko TP, Moore WM, Kasten TP, et al. Selective inhibition of the inducible nitric oxide synthase by aminoguanidine. Eur J Pharmacol 1993;233:119-25.

19. Mizuta T, Kawaguchi AT, Nakahara K, Kawashima Y. Simplified rat lung transplantation using cuff technique. J Thorac Cardiovasc Surg 1989;97:578-81.

20. Langrehr JM, Hoffman RA, Billiar TR, Lee KKW, Schraut WH, Simmons RL. Nitric oxide synthesis in the in vivo allograft response: a possible regulatory mechanism. Surgery 1991;110:335-42.

21. Misko TP, Schilling RJ, Salvemini D, Moore WM, Currie MG. A fluoronetric assay for the measurement of nitrite in biological samples. Anal Biochem 1993; 214:11-6.

22. Prop J, Ehrie MG, Capro JD, et al. Reimplantation response in isografted rat lung: analysis of causal factors. J ThORAC CARDIOVASC SURG 1984;87:702-11.

23. Shiraishi T, Mizuta T, DeMeester SR, et al. Ischemic injury does not increase rejection in rat lung allografts. Surg Forum 1994;45:273-6.

24. Kondo T, Marchevsky AM, Prehn J, Matloff JM, Waters PF, Jordan SC. Evidence of complete tolerance in a model of rat lung allografts. Transplantation 1991;52:928-30.

25. Yousem SA, Berry GJ, Brunt EM, et al. A working formulation for the standardization of nomenclature in the diagnosis of heart and lung rejection: Lung Rejection Study Group. J Heart Lung Transplant 1990;9:593-601.

26. Prop J, Wildevuur CRH, Nieuwenhuis P. Lung allograft rejection in the rat. Transplantation 1985;40: 126-31.

27. Langrehr JM, White DA, Hoffman RA, Simmons RL. Macrophages produce nitric oxide at allograft sites. Ann Surg 1993;218:159-66.

28. James SL, Glaven J. Macrophage cytotoxicity against schistosomula of Schistosoma mansoni involves arginine-dependent production of reactive nitrogen intermediates. J Immunol 1989;143:4208-12.

29. Vicendeau P, Daulouede S, Veyret B, Darde ML, Bouteille B, Lemesre JL. Nitric oxide mediated cytostatic activity on Trypanosoma brucei gambiense and Trypanosoma brucei brucei. Exp Parasitol 1992;75: 353-60.

30. Adams LB, Hibbs JB Jr, Taintor RR, Krahenbuhl JL. Microbiostatic effect of murine-activated macrophages for Taxoplasma gondii: role for the synthesis of inorganic nitrogen oxides from I-arginine. J Immunol 1990;144: 2725-9.
31. Adams LB, Franzblau SG, Vavrin Z, Hibbs JB Jr, Krahenbuhl JL. L-Arginine-dependent macrophage effector functions inhibit metabolic activity of $\mathrm{Myco}$ bacterium leprae. J Immunol 1991;147:1642-6.

32. Hibbs JB Jr, Taintor RR, Vavrin Z, Rachlin EM. Nitric oxide: a cytotoxic activated macrophage affector molecule. Biochem Biophys Res Commun 1988; 157:87-94.

33. Hibbs JB Jr, Taintor RR, Vavrin Z. Macrophage cytotoxicity: role for $\mathrm{L}$-arginine deiminase and imino nitrogen oxidation to nitrite. Science 1987;235: 473-6.

34. Stuehr DJ, Nathan CF. Nitric oxide: a macrophage product responsible for cytostasis and respiratory inhibition in tumor target cells. J Exp Med 1989;169: 1543-55.

35. Ignarro LJ. Heme-dependent activation of guanylate cyclase by nitric oxide: a novel signal transduction mechanism. Blood Vessels 1991;28:67-73.

36. Lepoivre M, Flaman JM, Henry Y. Early loss of the tryosyl radical in ribonucleotide reductase of adenocarcinoma cells producing nitric oxide. J Biol Chem 1992;267:22994-3000.

37. Hoffman RA, Langrehr JM, Billiar TR, Curran RD, Simmons RL. Alloantigen-induced activation of rat splenocytes is regulated by the oxidative metabolism of L-arginine. J Immunol 1990;145:2220-6.

38. Langrehr JM, Muller AR, Markus PM, Simmons RL, Hoffman RA. FK 506 inhibits nitric oxide production by cell infiltrating sponge matrix allografts. Transplant Proc 1991;23:3260-1.

39. Langrehr JM, Dull KE, Ochoa JB, et al. Evidence that nitric oxide production by in vivo allosensitized cells inhibits the development of allospecific CTL. Transplantation 1992;53:632-40.

40. Hoffman RA, Langrehr JM, Dull KE, Simmons RL. Nitric oxide production by mouse sponge matrix allograft-infiltrating cells. Transplantation 1993;55: 591-6.

41. Albina JE, Henry WL Jr. Suppression of lymphocyte proliferation through the nitric oxide synthesizing pathway. J Surg Res 1991;50:403-9.

42. Albina JE, Caldwell MD, Henry WL, Mills CD. Regulation of macrophage functions by L-arginine. $\mathbf{J}$ Exp Med 1989;169:1021-9.

43. Albina JE, Mills CD, Henry WL Jr, Caldwell MD. Regulation of macrophage physiology by L-arginine: role of the oxidative L-arginine deiminase pathway. $\mathbf{J}$ Immunol 1989;143:3641-6.

44. Ettinger SL, McLoughlin MG, Scudamore CH, Miller RR, Keown PA. Effects of recovery from ischemic injury on class I and class II MHC antigen. Transplantation 1990;49:641-3.

45. Adoumie R, Serrick C, Giaid A, et al. Early cellular events in the lung allograft. Ann Thorac Surg 1992; 54:1071-7. 


\section{Discussion}

Dr. Robert J. Keenan (Pittsburgh, Pa.). This is a welldesigned study that builds on previous work at Washington University on the role of NO synthase inhibition on modulation of cardiac allograft rejection. Dr. Shiraishi's research provides further evidence of the central role of NO in inflammatory processes.

The authors present data suggesting that NO production, as measured by serum nitrite/nitrate levels, is accelerated during early allograft rejection and that inhibition of inducible NO synthase by aminoguanidine ameliorates this activity and suppresses the rejection response. The evidence for this effect is based on histologic and radiographic criteria. In the written discussion the authors suggest that NO directly damages the allograft in the early posttransplantation period and that this damage leads to increased antigen expression and an enhanced allograft response. The paper does not present much data to support that hypothesis.

I have three questions. First, why did you wait until postoperative day 4 to assess NO production inasmuch as the peak of NO activity may well have been earlier in the process and you may have been catching this activity on the down slope?

Second, aminoguanidine, although a much more potent inhibitor of inducible NO synthase, does in fact have some activity against constitutive NO synthase activity. Did you look at alternative methods for detection of inducible NO synthase such as Northern Blot analysis or, more particularly, immunostaining to confirm that the effect you saw was truly due to inducible NO synthase inhibition?

Third, have you done any characterization of the cellular infiltrate and its time course over the rejection process to establish whether inducible NO synthase inhibition interferes with entry of circulating allograft cells into the graft or with inhibition of lymphocyte proliferation once those cells have entered the lung?

Dr. Shiraishi. Thank you, Dr. Keenan. You asked why we did not measure nitrite and the nitrate in the early period. The first paper concerning the increased production of NO was presented by Dr. Rambla and the group at Pittsburgh University. Using a wholly allogenic rat heart and liver and a small interesting transplant model, they demonstrated increased nitrate and nitrite levels in serum in the process of rejection. They started their assessments on day 3 , and there was a very small but significant difference. Because this difference became bigger associated with rejection, we decided to start my assessment from day 4.

This question includes the possibility that NO is produced in association with ischemic-reperfusion injury, so we probably could get more useful and additional information about NO metabolism, not only as a marker of ischemic-reperfusion injury, but also as an earlier marker of rejection.

The second question concerned whether aminoguanidine is an inducible NO synthase inhibitor. Unfortunately, we did not do any immunohistochemical staining for this study, so we have no clear answer.

The third question concerns the mechanism of NO in the rejection process. We have two hypotheses for the role of NO in the rejection process. One is its cytotoxic effect. NO was first demonstrated to be more cytotoxic against tumor cell line or pathogen or transplanted islet cells. Such injury may increase or up-regulate the autogenicity of transplanted organs. It may cause a high potential of rejection. The other possibility is its lymphoproliferative effects. We believe such potential will increase a tendency for rejection, but in this particular study we did not do any mechanical study, so we have no answer. 